\title{
Article \\ Efficient Recycling Blast Furnace Slag by Constructing Ti-Embedded Layered Double Hydroxide as Visible-Light-Driven Photocatalyst
}

\author{
Ningning Song, Yongfeng Cai $\mathbb{D}^{\mathrm{D}}$, Lingmin Sun, Peng $\mathrm{Hu}$ *, Qinqin Zhou, Junshu Wu and Jinshu Wang * \\ Key Laboratory of Advanced Functional Materials, Education Ministry of China, Faculty of Materials and \\ Manufacture, Beijing University of Technology, Beijing 100124, China; snn@emails.bjut.edu.cn (N.S.); \\ caiyf0127@emails.bjut.edu.cn (Y.C.); 13053593786@163.com (L.S.); zhouqinqin@njut.edu.cn (Q.Z.); \\ junshuwu@bjut.edu.cn (J.W.) \\ * Correspondence: pengh@bjut.edu.cn (P.H.); wangjsh@bjut.edu.cn (J.W.)
}

Citation: Song, N.; Cai, Y.; Sun, L.; $\mathrm{Hu}$, P.; Zhou, Q.; Wu, J.; Wang, J. Efficient Recycling Blast Furnace Slag by Constructing Ti-Embedded Layered Double Hydroxide as Visible-Light-Driven Photocatalyst. Materials 2022, 15, 1514. https:// doi.org/10.3390/ma15041514 Academic Editor: Alina Pruna Received: 20 January 2022 Accepted: 15 February 2022 Published: 17 February 2022

Publisher's Note: MDPI stays neutral with regard to jurisdictional claims in published maps and institutional affiliations.

Copyright: (C) 2022 by the authors. Licensee MDPI, Basel, Switzerland. This article is an open access article distributed under the terms and conditions of the Creative Commons Attribution (CC BY) license (https:// creativecommons.org/licenses/by/ $4.0 /)$.

\begin{abstract}
In this work, a strategy of heat treatment-precipitation has been developed to recycle Ti-containing metallurgical solid waste by forming Ti-embedded MgAl layered double hydroxide (TMA-LDH). This facile and simple route is featured by the dedicated utilization of the composition of slag with high overall recovery efficiency. Importantly, as-obtained product exhibits visible light response distinctly different from that of pristine MA-LDH ascribed to the Fe doping inherited from initial slag. Its mesoporous nanostructure also provides more microchannels for mass and carrier transfer. As such, excellent photocatalytic activity towards degradation of tetracycline hydrochloride is achieved, and $88 \%$ removal could be obtained in $60 \mathrm{~min}$. Furthermore, $44 \%$ increase in efficiency than that of Ti-excluded LDH also indicates the synergistically promoting effect of Ti incorporation. Mechanism investigation suggests that $\mathrm{Ti}$ incorporation regulates the electronic structure of pristine $\mathrm{LDH}$ with more active sites, and favors the formation of radicals with improved oxidative ability for photocatalysis.
\end{abstract}

Keywords: titanium-containing blast furnace slag; layered double hydroxide; Ti-embedding; visible light response; photocatalysis

\section{Introduction}

Titanium-containing blast furnace slag (Ti-BFS) is a by-product of the iron smelting process as metallurgical solid waste, and mainly composed of perovskite and aluminosilicates of calcium and magnesium, respectively [1]. Because of the large consumption of iron in modern society, the yield of Ti-BFS is quite huge and more than 3 million tons accumulates every year [2]. However, Ti-BFS with low content of Ti (10-25 wt. \%) is hard to utilize as raw material directly for Ti manufacture. Concentrating Ti by previously developed strategies, such as acid leaching [3], alkali molten salt calcining [4], carbonizationchlorination [5] and high-temperature enrichment [6], suffers from low efficiency and high cost. Currently, the mostly available route for Ti-BFS utilization is used as a whole for building materials considering its high content of $\mathrm{CaO}$ and $\mathrm{SiO}_{2}$, but excessive $\mathrm{TiO}_{2}(>10 \%)$ in Ti-BFS would suppress the polymerization degree of building materials and result in a negative effect on its volume stability [7]. As such, most of the Ti-BFS is disposed directly without further treatment, which causes the waste of Ti-containing secondary resource and poses a risk to the environment; thus, developing facile and efficient approaches to recycle Ti-BFS has become a critical issue needing to be seriously considered.

Layered double hydroxide ( $\mathrm{LDH})$ is a type of two-dimensional inorganic compound, in which bivalent (such as $\mathrm{Mg}^{2+}, \mathrm{Zn}^{2+}, \mathrm{Ni}^{2+}$ ) and trivalent metallic cations (such as $\mathrm{Al}^{3+}, \mathrm{Fe}^{3+}$, $\mathrm{In}^{3+}$ ) construct the layered framework, while exchangeable anions occupy the interlayer region for charge compensation [8]. Owing to its unique structure and synergistic effect 
of bimetals, $\mathrm{LDH}$ is endowed with exceptional properties, and has been widely applied in adsorbents $[9,10]$, catalysts [11,12], energy storage [13,14], drug delivery [15,16], etc. Specially, LDH possesses abundant hydroxyl groups, which provides potential active hydroxyl radicals to break the ring structure of organic compounds (such as toluene, TC) excited by light irradiation. In addition, the typically sheet-like structure also provides high surface to volume ratio, and enriches the active sites on the surface to further promote the photocatalytic reaction. As such, LDH exhibits great potential as efficient photocatalyst for organic pollutant degradation. Up to now, various routes have been developed for LDH preparation, such as co-precipitation, hydrothermal, anion exchange and sol-gel methods [17-20]. Among these methods, alkali-precipitation has attracted much attention due to its simple and low-cost features, and brings LDHs closer to practical applications. Considering the existence of bivalent $\mathrm{Mg}$ and trivalent $\mathrm{Al}$ in Ti-BFS with high content, it is reasonable to expect that Ti-BFS can be served as potential raw material for MgAl-LDH preparation, which not only achieves the valuable utilization of Ti-BFS, but also decreases the cost of LDH. More recently, Xiao et al. explored the synthesis of CaFeAl-LDH by using calcium carbide slag and red mud composites as raw material, and the as-obtained product exhibits high ability for phosphorus adsorption [21]. However, the thermal treatment of calcium carbide possibly causes the formation of harmful carbon monoxide or carbon oxide gas, and detailed recovery efficiency has not been investigated in their work. As such, further work still needs to be conducted to properly address the facile and mild synthesis of LDH from metallurgical waste with acceptable extraction efficiency, and provide more promising recycling strategies for Ti-BFS.

In this work, a heat treatment-precipitation strategy was developed to recycle Ti-BFS efficiently by formation of products including the Si-Ca compound and Ti-embedded MgAl-LDH, respectively. The deliberate utilization of the slag mixture emphasizes the overall recovery efficiency of almost over $90 \%$. Furthermore, the photocatalytic activity of as-obtained MgAl-LDH was evaluated by removal of tetracycline under visible light irradiation, and superior performance was identified by $92 \%$ degradation of initial concentration. In addition, enhanced light absorption and synergistic effect of $\mathrm{Ti}$ that contributed to the promoted organic pollutant removal were also investigated in detail.

\section{Materials and Methods}

\subsection{Materials}

The Ti-BFS was obtained from Pan Steel Group in Sichuan, China, and the main composition is given in Table 1. All the other chemicals are of analytical grade and used as received without further purification.

Table 1. The main composition of Ti-BFS.

\begin{tabular}{cccccc}
\hline Composition & $\mathbf{C a O}$ & $\mathbf{S i O}_{2}$ & $\mathrm{TiO}_{2}$ & $\mathbf{M g O}$ & $\mathbf{A l}_{2} \mathbf{O}_{3}$ \\
\hline wt. $\%$ & 28.91 & 26.16 & 21.45 & 9.05 & 14.43 \\
\hline
\end{tabular}

\subsection{Recycling Process of Ti-BFS}

The schematic illustration of the recycling process of Ti-BFS is presented in Figure 1. Firstly, Ti-BFS was grinded by ball milling and sieved with 80 mesh screen, and $5 \mathrm{~g}$ fine Ti-BFS was mixed with $40 \mathrm{~g}$ ammonium sulfate followed by thermal treatment at $450{ }^{\circ} \mathrm{C}$ for $1 \mathrm{~h}$. After reaction, the product was immersed into $160 \mathrm{~mL}$ water at $80^{\circ} \mathrm{C}$ for $2 \mathrm{~h}$, and the insoluble residue was separated by centrifugation and named as CSS. Then the $\mathrm{pH}$ of obtained supernatant was adjusted to $10 \mathrm{using} 2 \mathrm{~mol} / \mathrm{L} \mathrm{NaOH}$ solution, and aged at $70{ }^{\circ} \mathrm{C}$ for $4 \mathrm{~h}$ to obtain a suspension. Finally, Ti-embedded hydrotalcite product was collected by centrifugation and washed with deionized water and ethanol, respectively, and further dried at $60{ }^{\circ} \mathrm{C}$ in an oven. As obtained product was named as TMA-LDH. The optical images of the samples are shown in the inset pictures of Figure 1. 


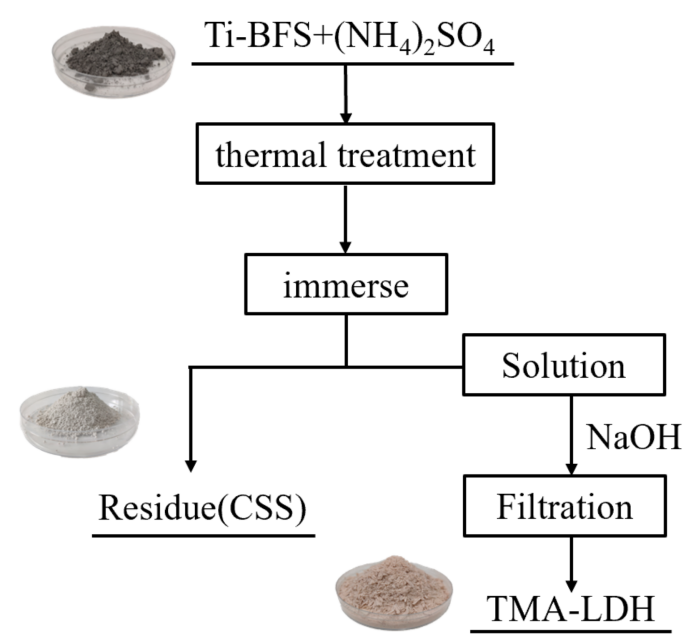

Figure 1. Schematic illustration of the recycling process of Ti-BFS.

The recovery efficiency was calculated according to the overall weight and contents of corresponding elements in initial slag, obtained CSS and TMA-LDH, respectively. Based on ICP analysis, the contents of component elements in different products could be estimated, and then the accurate weights were calculated by separating the overall weight according to their relative proportion. Finally, the recovery efficiency of different elements could be determined by their total amount in CSS and LDH compared to the values in initial slag.

To investigate the influence of $\mathrm{Ti}$ incorporation on the structure and property of obtained layered double hydroxide, a controlled experiment was conducted to exclude Ti in hydrotalcite by adjusting the $\mathrm{pH}$ of supernatant to 4 , which results in the formation of $\mathrm{TiOSO}_{4}$ precipitation. After filtration, the hydrotalcite without Ti was obtained by further adjusting the $\mathrm{pH}$ of supernatant to 10 , and as-obtained product was named as MA-LDH.

\subsection{Characterizations}

The phases of obtained products were investigated by powder X-ray diffraction patterns (XRD) on a Bruker D8 Advance diffractometer using $\mathrm{Cu}-\mathrm{K} \alpha$ radiation. The elemental composition was determined using inductively coupled plasma spectrometer (ICP, Optima 8300). Scanning electron microscopy (SEM, Hitachi SU8020) and transmission electron micrograph (TEM, FEI Talos F200X-G2) were adopted to investigate the morphologies of samples. The ASAP 2020 volumetric adsorption analyzer was used to measure the nitrogen adsorption-desorption isotherms at $77 \mathrm{~K}$ to analyze the specific surface area and average pore volume of the samples. Ultraviolet-visible diffuse reflectance spectroscopy was collected on Shimadzu UV2550 spectrophotometer. The X-ray photoelectron spectroscopy (XPS) spectrum was obtained by an ESCALAB250 X-ray photoelectron spectrometer to reveal the element composition and chemical state of the samples. All XPS peaks of the elements were calibrated against the $\mathrm{C} 1 \mathrm{~s}$ line fixed at $284.6 \mathrm{eV}$ as a reference. The ${ }^{27} \mathrm{Al}$ highresolution NMR spectra were obtained at $750 \mathrm{MHz}$ principal field on a Bruker AVANCE III equipped with high-speed MAS probe with $20 \mathrm{mg}$ samples. Electron paramagnetic resonance spectroscopy (EPR) spectra were used to detect the photo-excited active species during photocatalytic reaction on a Bruker EMX spectrometer with $400 \mathrm{mg} / \mathrm{L}$ solution.

\subsection{Photocatalytic Activity Measurement}

Photocatalytic activity of the samples was characterized by degradation of tetracycline hydrochloride (TC) under visible light irradiation. A Xe lamp (400 W, Perfect Light) with a cut-off filter of $420 \mathrm{~nm}$ was used as light source. In a typical photocatalytic process, 5-25 mg of obtained LDH and $50 \mathrm{~mL}$ of 5-100 mg/L TC solution were mixed for photocatalytic reaction, and the suspension was firstly stirred in the dark for $30 \mathrm{~min}$ to achieve nearly full adsorption-desorption equilibrium. At specific light illumination intervals, $3 \mathrm{~mL}$ of the reaction solution was taken and centrifuged to remove the catalyst sample and analyzed 
by UV-vis absorption spectroscopy. The residual TC concentration of filtrate was tested according to its absorbance at a wavelength of $375 \mathrm{~nm}$. After one cycle of test, the catalyst was collected by centrifugation, and washed with water and ethanol several times. Then, the catalyst was dried in an oven at $60{ }^{\circ} \mathrm{C}$ for $24 \mathrm{~h}$ for the next test. Three cycles of tests were conducted to investigate the stability of obtained catalyst.

\section{Results and Discussion}

In order to efficiently recover Ti-BFS, ammonium sulfate was firstly adopted to react with metal cations including $\mathrm{Ti}, \mathrm{Ca}, \mathrm{Mg}$ and $\mathrm{Al}$ in Ti-BFS, and corresponding sulfates generated during the thermal treatment process, which facilitates the elemental separation in the following immersing process considering their different solubility in water. Insoluble $\mathrm{CaSO}_{4}$ and unreacted $\mathrm{SiO}_{2}$ entered solid residue, and can be used as raw materials for cement. Soluble $\mathrm{Ti}, \mathrm{Mg}$ and $\mathrm{Al}$ cations were further precipitated by adjusting the $\mathrm{pH}$ of the solution to alkaline state, which results in the formation of Ti embedded MgAl-LDH. The reaction mechanism could be verified by phase evolution of slag during recycling process as illustrated in Figure 2. Compared to the dominated perovskite phase and aluminosilicates of calcium and magnesium phases in pristine Ti-BFS, as-obtained CSS was mainly composed of calcium dihydrate and metasilicic acid, respectively. While the exclusive phase of hydrotalcite-like MgAl-LDH was observed in precipitated TMA-LDH and MA-LDH products, illustrating the formation of layered double hydroxide. In addition, the absence of Ti-related phases in TMA-LDH was possibly ascribed to its efficient incorporation into the framework of LDHs. This feasible alkali precipitation has also been widely adopted to synthesize pristine and doped LDHs in previous work [22-25].

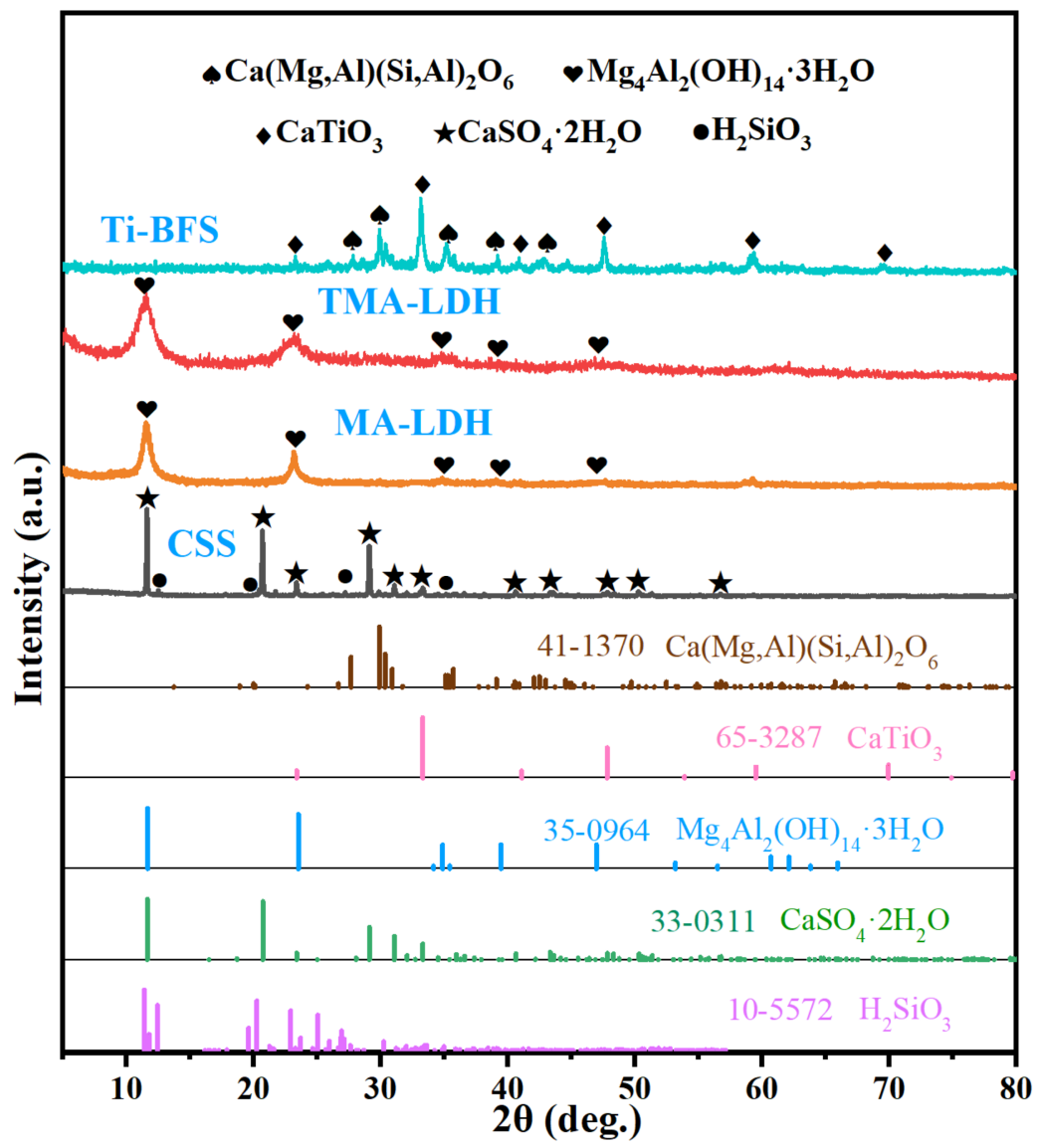

Figure 2. XRD patterns of Ti-BFS and obtained various products.

Furthermore, composition analysis gives quantitative information of element distribution in the obtained products. As illustrated in Table 2, Si and Ca in CSS were identified 
with weight ratio higher than $90 \%$, indicating good separation and enrichment of Si and Ca during calcination and the immersion process. While the left elements were mostly preserved in $\mathrm{LDH}$, and the existence of $\mathrm{Ti}$ in TMA-LDH was also confirmed by composition analysis. It should be noted that sharp decrease of Ti content in obtained MA-LDH could also be identified by elemental analysis as shown in Table S1. In addition, the general weight ratios of main elements converted in CSS and TMA-LDH are listed in Table S2. As indicated, the overall recovery efficiency beyond $90 \%$ could be achieved for $\mathrm{Si}, \mathrm{Ti}, \mathrm{Al}$ and $\mathrm{Mg}$, respectively. Furthermore, above results indicate the feasibility of here developed method as a promising strategy for Ti-BFS recycling with high efficiency and additional value.

Table 2. Element distribution in different products and corresponding recovery efficiency.

\begin{tabular}{cccc}
\hline $\begin{array}{c}\text { Compositional } \\
\text { Element }\end{array}$ & CSS (wt. \%) & TMA-LDH (wt. \%) & $\begin{array}{c}\text { Recovery Efficiency } \\
\text { (wt. \%) }\end{array}$ \\
\hline $\mathrm{Ca}$ & 55.67 & 5.71 & 88.11 \\
$\mathrm{Si}$ & 37.59 & 1.74 & 96.00 \\
$\mathrm{Ti}$ & 5.30 & 52.19 & 95.26 \\
$\mathrm{Al}$ & 0.05 & 17.83 & 97.14 \\
$\mathrm{Mg}$ & 1.39 & 22.53 & 91.87 \\
\hline
\end{tabular}

The morphology of synthesized TMA-LDH was investigated by electron microscope observation. As indicated in Figure 3a, flower-like particles with size of about $100 \mathrm{~nm}$ dominated in the obtained product, and the magnified SEM image in Figure $3 \mathrm{~b}$ further reveals that the particle was assembled by loosely aggregated nanosheets as building blocks. The detailed structure was also identified by TEM in Figure 3c, and it was observed that assembled nanosheets were connected to each other by the formation of a net-like structure. Enlarged TEM images further illustrate the thinness of the nanosheet according to the brightness contrast between the sample and the substrate. Furthermore, EDS mapping was adopted to visually describe the elemental distribution of $\mathrm{LDH}$, and $\mathrm{Al}, \mathrm{Mg}$, $\mathrm{Ti}$ and $\mathrm{O}$ were well distributed in the product. Considering the uniform morphology and homogeneous elemental distribution, it is suggested that $\mathrm{Ti}$ is incorporated into the framework of layered double hydroxide. In addition, the morphology of MA-LDH was also investigated as shown in Figure S1. As indicated, sheet-like nanostructures with less aggregation were observed, which illustrates the shape evolution induced by sharply decreased Ti content.
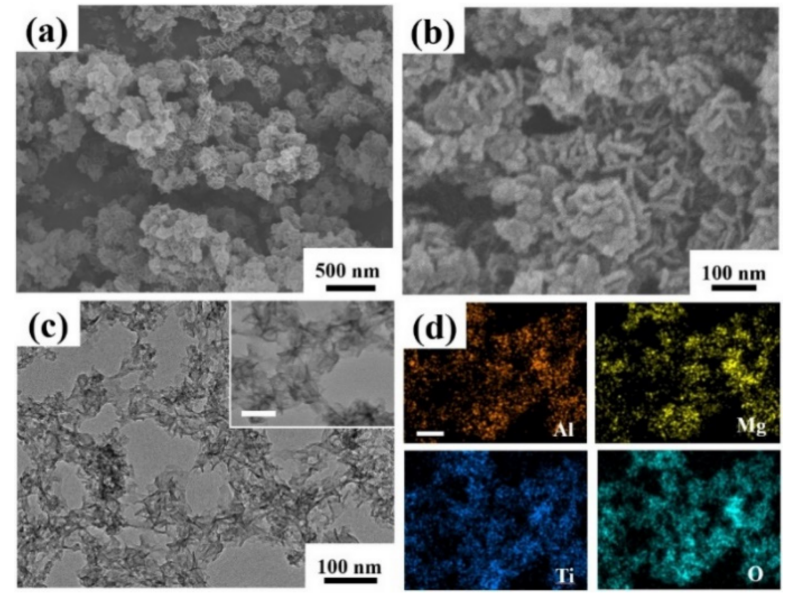

Figure 3. (a) Low and (b) high magnified SEM images, (c) TEM images and (d) elemental mapping of obtained TMA-LDH. The scale bars of inserted images in c and d are $50 \mathrm{~nm}$.

The structure and property of obtained brucite products were further investigated as shown in Figure 4. FT-IR spectra were firstly adopted to identify the functional groups of synthesized layered double hydroxides as shown in Figure $4 \mathrm{a}$, and the similar functional 
groups were presented both in MA-LDH and TMA-LDH, respectively. In the case of TMA-LDH, typically stretching vibration of the hydroxyl groups is observed at the band of about $3409 \mathrm{~cm}^{-1}$, which is distinct from the vibration of $\mathrm{H}_{2} \mathrm{O}$ molecules at $1629 \mathrm{~cm}^{-1}$ [26]. In addition, the peaks at $798 \mathrm{~cm}^{-1}$ and $670 \mathrm{~cm}^{-1}$ are ascribed to the typical vibrations of $\mathrm{Al}-\mathrm{OH}$ and $\mathrm{Mg}-\mathrm{OH}$ bonds in $\mathrm{MgAl} \mathrm{LDH}$, respectively [27]. The bonding structure of Ti was recognized as an $\mathrm{O}-\mathrm{Ti}-\mathrm{O}$ bond with the vibration peak at $483 \mathrm{~cm}^{-1}$ [28]. Furthermore, a fresh generated peak at $580 \mathrm{~cm}^{-1}$ was recognized and attributed to the Fe-O vibration [29], and this result illustrates the existence of Fe in as-obtained product, which is possibly originated from the residual Fe in pristine Ti-BFS.
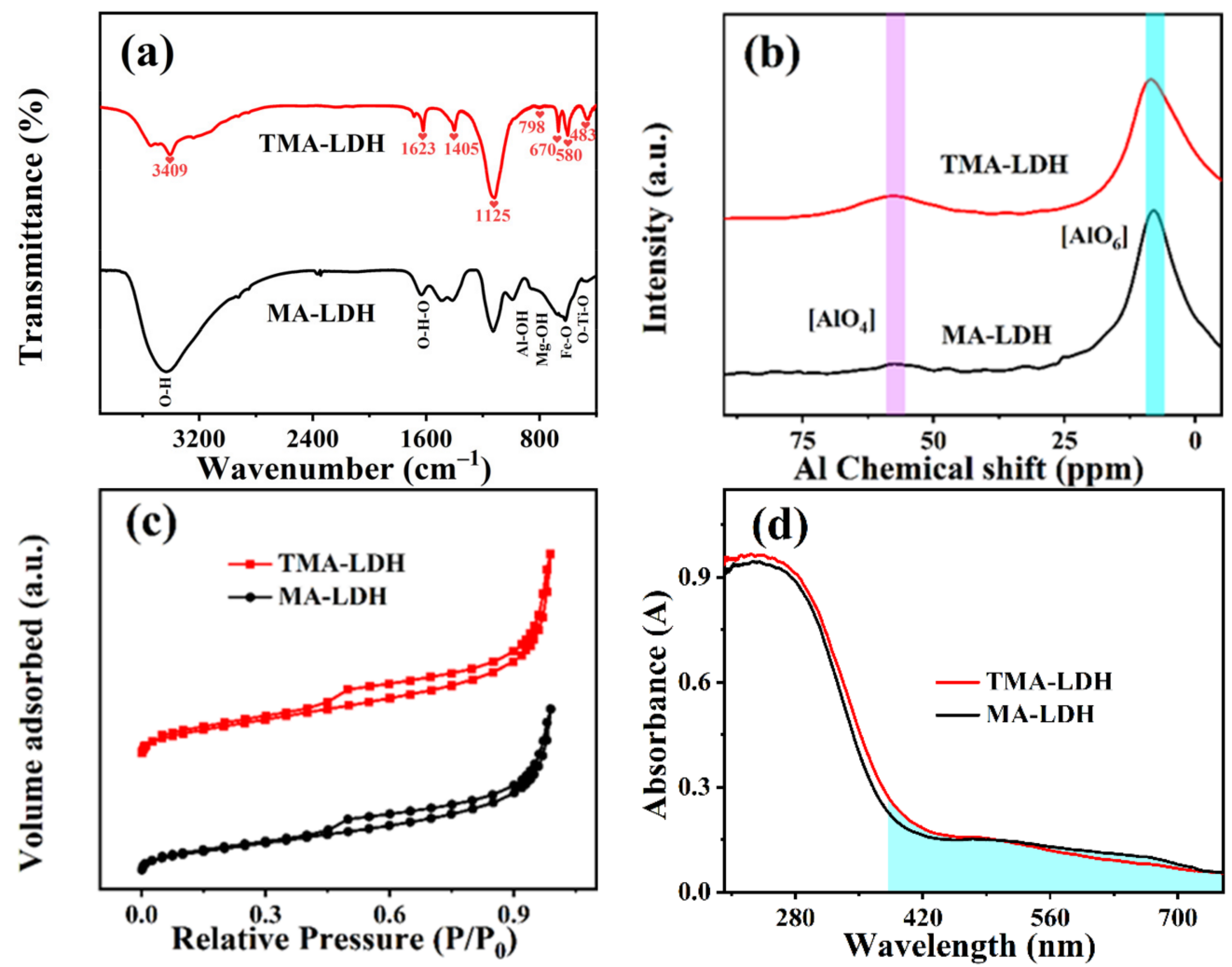

Figure 4. (a) FT-IR spectra, (b) NMR spectroscopy, (c) nitrogen adsorption-desorption isotherm and (d) UV-vis absorption spectra of synthesized brucite products.

Layered structure of LDH is featured by the alternatively arranged metals coordinated to six hydroxyl groups in an octahedral geometry, thus six-coordinated (octahedral) $\mathrm{AlO}_{6}$ is the typical structure unit of $\mathrm{MgAl} \mathrm{LDH} \mathrm{[30].} \mathrm{This} \mathrm{could} \mathrm{be} \mathrm{verified} \mathrm{well} \mathrm{by} \mathrm{the} \mathrm{strong}$ signal of $\mathrm{AlO}_{6}$ resonance in NMR spectroscopy shown in Figure $4 \mathrm{~b}$. In addition, a weak peak ascribed to four-coordinated $\mathrm{AlO}_{4}$ was also observed, suggesting the crystallographic change of aluminum in octahedral positions, and possibly attributed to the structural distortion by $\mathrm{Ti}$ containing within the octahedral $\mathrm{TiO}_{6}$ configuration [31]. Importantly, decreased $\mathrm{AlO}_{6}$ signal and increased $\mathrm{AlO}_{4}$ signal in TMA-LDH also illustrates the stronger structural distortion due to its rather high Ti content than that of MA-LDH.

The specific surface area of obtained LDHs was investigated by nitrogen adsorptiondesorption isotherm. Type IV isotherms are well presented in Figure 4c, indicating the mesoporous structure of the two products. The specific surface area was further determined to be about $135.76 \mathrm{~m}^{2} / \mathrm{g}$ and $188.44 \mathrm{~m}^{2} / \mathrm{g}$, together with similar pore sizes of $3.8 \mathrm{~nm}$ for TMA-LDH and MA-LDH (Figure S2), respectively. This result suggests the negligible effect of Ti content on the specific surface area of the obtained products. Notably, high surface area with porous structure provides more microchannels for mass transport and active sites for catalytic reaction, and in turn promotes the photocatalytic performance due to 
the enhanced kinetic nature. Figure $4 \mathrm{~d}$ gives the UV-vis absorption spectra of TMA-LDH and MA-LDH, and similar curves were identified. Impressively, high absorption shoulder with red-shift absorption edge was observed for two samples, suggesting their enhanced light response of visible light absorption. According to the UV-vis curves, the bandgap energy could be calculated by the Tauc plots shown in Figure S4, and the values are $2.71 \mathrm{eV}$ and $2.79 \mathrm{eV}$ for TMA-LDH and MA-LDH, respectively. It is worth noting that pristine MgAl-LDH can only absorb ultraviolet light [8], which suppresses the efficient utilization of solar energy by more than 50\% energy in the visible light zone. Thus, the enhanced ability of light absorption illustrates more available protons excited by light, and is favorable to the following redox reaction. Considering the existence of Fe in obtained LDH and initial slag (Figure S3), it is reasonably speculated that the enhanced visible light absorption is originated from the Fe doping [32].

The chemical composition and valence state on the near surface of as-synthesized LDHs were investigated by XPS analysis. All XPS peaks of the composition elements were calibrated against the C 1s line fixed at $284.6 \mathrm{eV}$ (XPS spectra of C are given in Figure S5). According to the overview measurement in Figure $5 \mathrm{a}, \mathrm{Al}, \mathrm{Mg}$, Ti and $\mathrm{O}$ could be detected both in TMA-LDH and MA-LDH, respectively. Figure 5b presents the high-resolution spectra of $\mathrm{Al} 2 \mathrm{p}$, and the peak located at $74.8 \mathrm{eV}$ could be attributed to the Al-OH structure in the brucite-like layer of MA-LDH [33]. Apparently, the band energy of $\mathrm{Al} 2 \mathrm{p}$ was increased to $75.0 \mathrm{eV}$ for TMA-LDH, indicating the enhanced electron density around $\mathrm{Al}$ sites $[34,35]$. In addition, the binding energy of $\mathrm{Mg} 1 \mathrm{~s}$ in Figure $5 \mathrm{c}$ reveals its chemical states of oxide/hydroxide at $1035.3 \mathrm{eV}$ in MA-LDH [36]. The decreased binding energy of $0.3 \mathrm{eV}$ for TMA-LDH suggests the possible electron transfer from $\mathrm{Mg}$ to $\mathrm{Al}$ after Ti incorporation, and results in redistribution of spatial electron density around $\mathrm{Mg}$ and $\mathrm{Al}$ sites, respectively. Because the intrinsic electronic structure directly determines the surface chemical behavior that is highly related to the catalytic properties; adjustable photocatalytic performance is expected by modulating MA-LDH through Ti incorporation.

Ti $2 p$ spectra of TMA-LDH and MA-LDH exhibit two separate peaks assigned to the $\mathrm{Ti}^{4+} 2 \mathrm{p}_{3 / 2}$ and $\mathrm{Ti}^{4+} 2 \mathrm{p}_{1 / 2}$ [37], respectively (Figure $5 \mathrm{~d}$ ), but the peak intensity is quite different for two samples. The rather strong intensity of TMA-LDH indicates its high content of $\mathrm{Ti}$, which is well in accordance with the composition analysis. In addition, the increased binding energy of $\mathrm{Ti}^{4+} 2 \mathrm{p}_{3 / 2}$ and $\mathrm{Ti}^{4+} 2 \mathrm{p}_{1 / 2}$ in TMA-LDH could also be observed, illustrating the effective Ti embedding in the framework of LDH. XPS fitting of Fe $2 p$ (Figure 5e) is to provide a qualitative illustration of iron on the near surface of LDHs. Two peaks with the binding energy of $713.60 \mathrm{eV}$ and $727.00 \mathrm{eV}$ could be recognized and well assigned to Fe $2 \mathrm{p}_{3 / 2}$ and Fe $2 \mathrm{p}_{1 / 2}$, respectively [38,39]. The detailed Fe content is determined to be about $0.5 \mathrm{wt}$. \% by ICP analysis. Figure $5 \mathrm{f}$ gives the core level spectrum of $\mathrm{O} 1 \mathrm{~s}$, and two fitted peaks with binding energy of about $532.3 \mathrm{eV}$ and $530.2 \mathrm{eV}$ could be observed, which are ascribed to the hydroxyl groups and adsorbed oxygen species [40], respectively. It should be noted that $\mathrm{Ti}$ incorporation also increases the electron density around the $\mathrm{O}$ site, which is clearly indicated by the positive shift of its energy band, possibly due to the crystallographic change as illustrated in Figure 5b.

Considering the high surface area and enhanced light absorption ability, as-obtained $\mathrm{LDH}$ is expected to present high performance in photocatalysis. Here, the degradation behaviors of TC over different catalysts were investigated under visible light $(\lambda>420 \mathrm{~nm})$, and presented in Figure 6a. As indicated, Ti-BFS, MA-LDH and TMA-LDH achieve adsorptiondesorption equilibrium after 90 min stirring in the dark, and the adsorption efficiency is determined to be about $8.11 \%, 8.85 \%$ and $9.31 \%$, respectively. Before photocatalytic testing, 30 min stirring was maintained in the dark to achieve nearly full adsorption-desorption equilibrium, and the adsorption efficiencies are about $5.40 \%, 6.11 \%$ and $6.42 \%$, respectively. According to the degradation curve, Ti-BFS shows rather low photocatalytic activity, and only 30\% TC was degraded after reaction for $60 \mathrm{~min}$. With the introduction of MA-LDH as photocatalyst, the degradation efficiency was significantly enhanced to $61 \%$. Importantly, rather low TC concentration of $12 \%$ was further obtained when adopting TMA-LDH as 
catalyst. The degradation efficiency is about 2.9 times higher than that of Ti-BFS, and $44 \%$ increase than that of MA-LDH, which illustrates the remarkably promoting effect well of Ti on the photocatalytic activity of obtained LDH. It should be noted that TC photolysis presents negligible contribution on the degradation efficiency of photocatalysts.
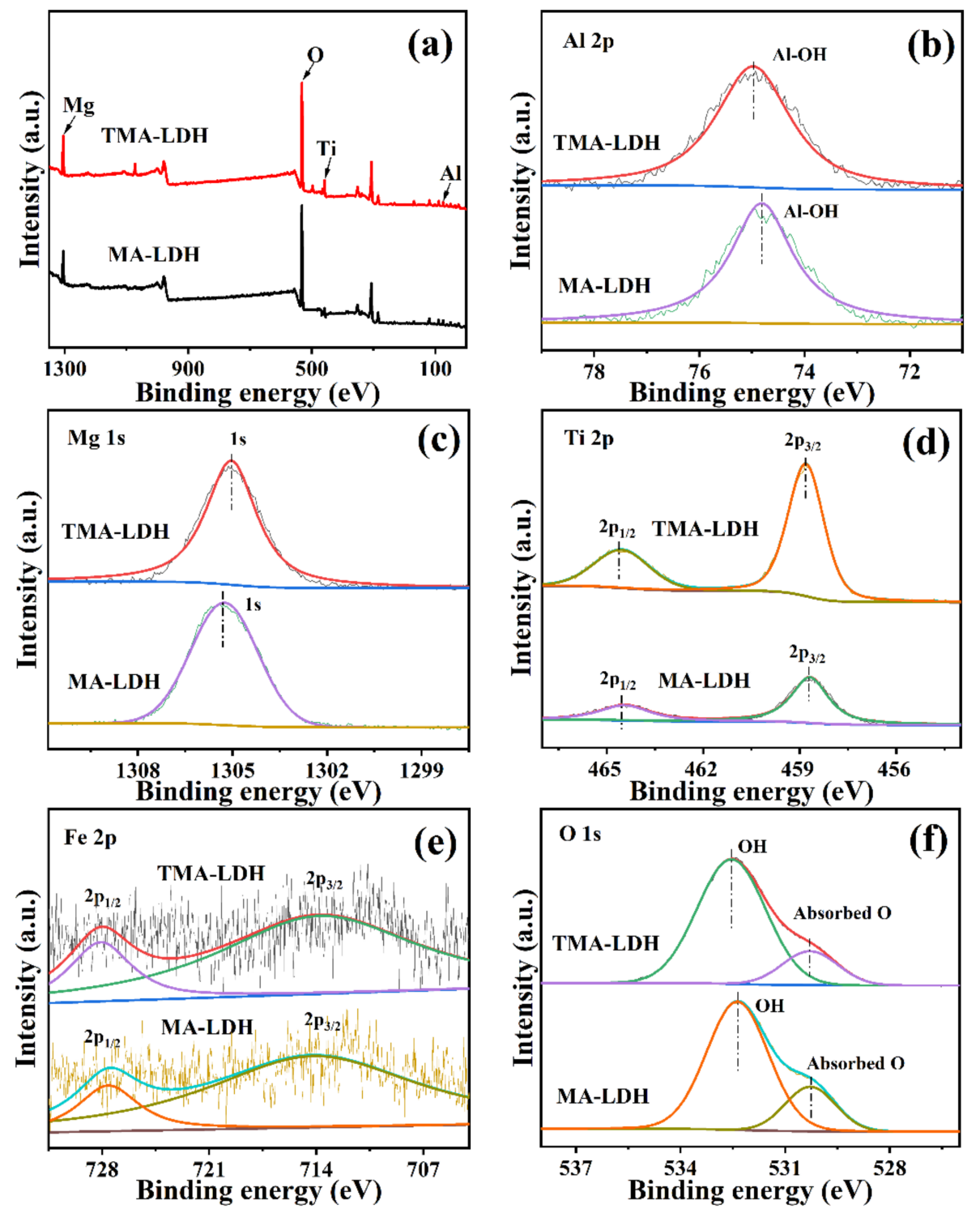

Figure 5. XPS spectra of obtained TMA-LDH and MA-LDH: (a)survey, (b) Al 2p, (c) Mg 1s, (d) Ti 2p, (e) Fe $2 \mathrm{p}$ and (f) $\mathrm{O} 1 \mathrm{~s}$.

Figure $6 \mathrm{~b}$ gives the influence of catalyst dosage on the degradation efficiency of TC. Upon increasing the amount of $\mathrm{LDH}$, the photocatalytic activity was firstly enhanced, and reached the highest value at $0.4 \mathrm{~g} / \mathrm{L}$. When the usage of catalyst exceeded $0.4 \mathrm{~g} / \mathrm{L}$, the degradation efficiency was further decreased. The reason is that excessive catalyst would shield the light transmittance in the solution, and insufficient photon flux was received for the catalysts far away from the light source, which hinders the catalytic reaction and suppresses the degradation efficiency. Similar results were also observed for photocatalytic tests conducted under different $\mathrm{pH}$ and TC concentrations, as illustrated in Figure $6 \mathrm{c}, \mathrm{d}$. 

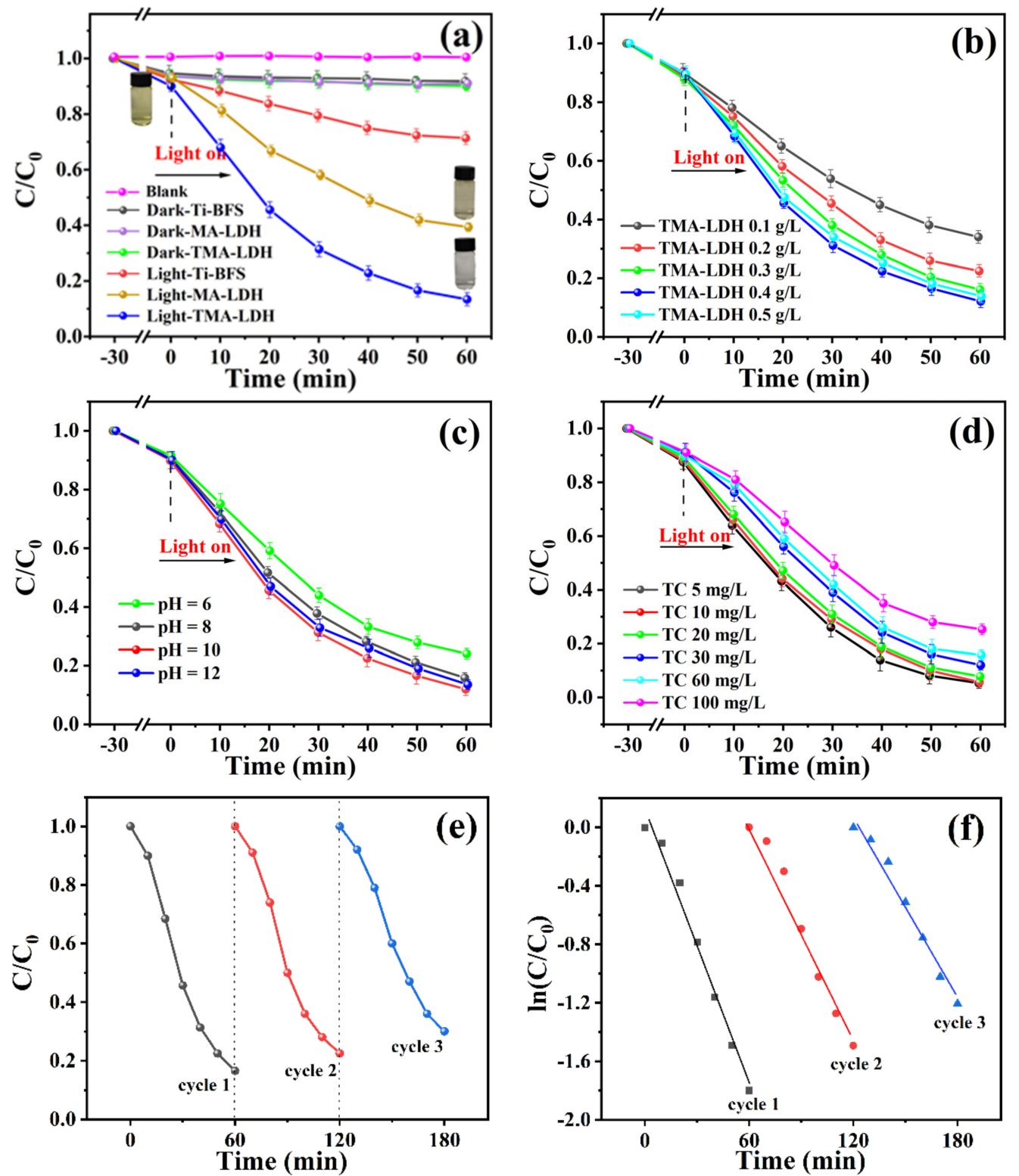

Figure 6. Photocatalytic performance of as-synthesized catalysts towards degradation of TC under visible light irradiation: (a) photo-degradation efficiency as function of reaction time; effect of (b) catalyst dosage, (c) initial $\mathrm{pH}$ value and (d) initial TC concentration on the photo-degradation efficiency of TC; (e) stability of TMA-LDH for TC degradation and (f) reaction kinetics analysis of three cycles of photocatalysis reaction.

To assess the stability and recyclability of TMA-LDH catalyst, three cycles of photocatalysis tests were conducted and the results are displayed in Figure 6e. Compared to $88 \%$ degradation of TC during the first cycle, $70 \%$ removal of initial TC could be obtained during the third cycle, indicating $18 \%$ decay of photocatalytic capacity after three cycles. Furthermore, the kinetic feature of photocatalyst over three cycles of reaction was also investigated as shown in Figure 6f. It is obvious that the photo-degradation rates can be well fitted to pseudo-first-order kinetics according to the equation of $\ln \left(C / C_{0}\right)=k_{a p p} t$, where $\mathrm{k}_{\mathrm{app}}$ represents the rate constant and $\mathrm{t}$ is the reaction time. Thus, $\mathrm{k}_{\mathrm{app}}$ of three cycles of reaction was identified to be about $0.030,0.024$ and 0.021 , respectively. The well-preserved $\mathrm{k}_{\mathrm{app}}$ with high values not only indicates the favorable kinetic feature, but also suggests the stable reaction rates during the cycled photocatalytic process. Thus, the feasibility of as-synthesized catalyst as potential candidate for practical applications was well illustrated. 
To obtain intrinsic understanding of the photocatalytic mechanism, radical capture experiments were designed to identify the active species during the reaction process. Scavengers including tertiary butanol (TBA), 1,4-benzoquinone (BQ), potassium bromate $\left(\mathrm{KBrO}_{3}\right)$ and ethylene diamine tetra acetic acid disodium salt (EDTA-2Na) were adopted to quench the hydroxyl radicals $(\cdot \mathrm{OH})$, superoxide radicals $\left(\mathrm{O}_{2}{ }^{-}\right)$, photogenerated electrons $\left(\mathrm{e}^{-}\right)$and holes $\left(\mathrm{h}^{+}\right)$, respectively. As indicated in Figure $7 \mathrm{a}, \mathrm{b}$, the degradation efficiency of TC was remarkably suppressed by adding TBA and EDTA-2Na, and the values were determined to be $30 \%$ and $39 \%$, respectively. While $69 \%$ decrease in photocatalytic efficiency was observed with existence of $\mathrm{BQ}$. However, addition of $\mathrm{KBrO}_{3}$ exhibited a slight effect on the degradation rate of TC. Thus, $\cdot \mathrm{OH}$ and $\mathrm{h}^{+}$excited on the valence band of TMA$\mathrm{LDH}$ were identified as the predominant active species rather than $\cdot \mathrm{O}_{2}{ }^{-}$and $\mathrm{e}^{-}$on the conduction band, and oxidation reaction was recognized as the main mechanism for the photocatalytic removal of TC over TMA-LDH catalyst.
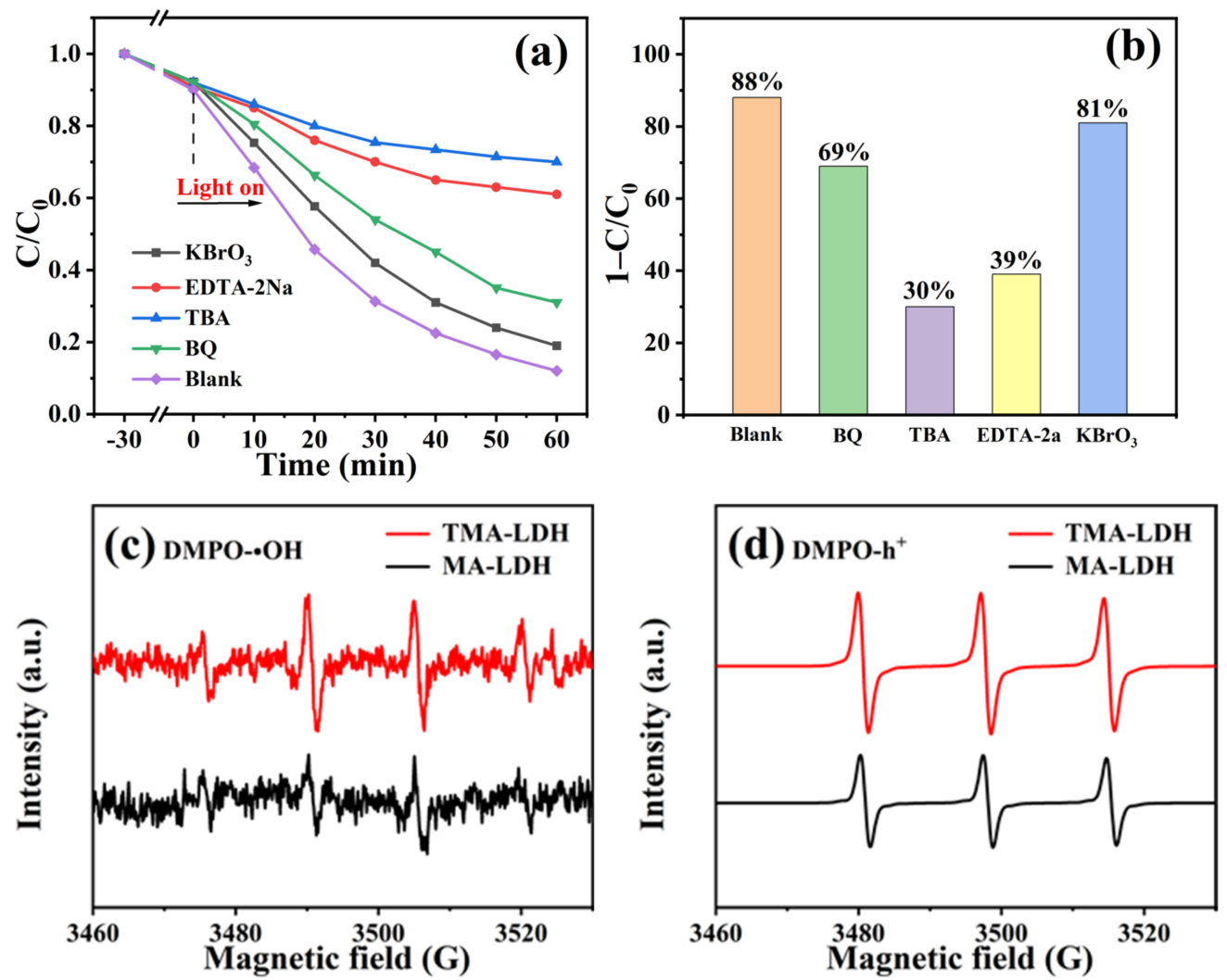

Figure 7. (a) Photo-degradation of TC and (b) comparison of degradation efficiency of TMA-LDH with presence of different radical scavengers, (c) EPR spectra of DMPO-.OH and (d) DMPO-h ${ }^{+}$of obtained TMA-LDH and MA-LDHs.

ESR spectra were also adopted to investigate the promoting mechanism of Ti incorporation on the photocatalytic activity of obtained product. As illustrated in Figure 7c, d, the signals of $\cdot \mathrm{OH}$ and $\mathrm{h}^{+}$under light irradiation were detected for two LDHs, which is well consistent with the results of the trapping experiments. Furthermore, relatively stronger signals both for $\cdot \mathrm{OH}$ and $\mathrm{h}^{+}$could be observed for MA-LDH, and suggests the generation of more active species under visible light irradiation. As such, it is reasonably speculated that Ti incorporation promotes the formation of active species during photocatalytic reaction, and is responsible for the greatly enhanced performance of LDH.

Heteroatom doping is an efficient strategy to regulate the electronic structure of semiconductors [41]. By partially destroying the periodicity of the crystal lattice, locally re-distributed electronic structure was achieved. This modification effectively changes the chemical behaviors of the reaction intermediates, and in turn enhances the catalytic ability 
of catalysts [34]. Previously theoretical and experimental work also reveal the feasibility of heteroatom doping on promoting the catalytic activity of LDHs [42]. Specially, Zhang et al. reported that high-valence state metal (Ta) caused the lattice expansion of LDH, and resulted in the modified $e_{g}$ orbital of Ta by directional charge transfer. Thus, Ta could serve as freshly generated active sites with preferred adsorption ability for intermediate species, and further improved the catalytic activity due to the enhanced reaction kinetics [43]. Furthermore, Kang et al. illustrated that the bonding nature for a mixed oxide structure of LDHs could be well adjusted by Ti embedding, and resulted Ni/Ti LDH exhibited high photocatalytic activity towards water oxidation [44]. In our work, Ti incorporation exhibited negligible effect on the surface area and light absorption, but the enhanced oxidation ability was achieved and ascribed to the increased active species of $\cdot \mathrm{OH}$ and $\mathrm{h}^{+}$ excited on the valence. As such, the modulated electronic structure is suggested to be the intrinsic mechanism for the improved photocatalytic performance, which is also confirmed by the structural investigations, such as XPS and NMR.

The degradation pathway of TC during photocatalysis has been investigated by many works. Firstly, the functional groups with high energy (such as amine groups and phenol groups) were interacted with active species and detached from TC molecules [45]. Then the hydrolyzed TC further lost its functional groups as the reaction proceeds, together with broken cyclic hydrocarbon structure [46]. After a series of redox reactions, the intermediates were mineralized and generated final products of $\mathrm{CO}_{2}$ and $\mathrm{H}_{2} \mathrm{O}$ [47]. Based on the above discussion, it is reasonable to summarize the mechanism of increased photocatalytic activity of TMA-LDH catalyst, and the schematic illustration is presented in Figure 8. Under irradiation with visible light, photo-induced holes can be excited to the valence band of catalyst and further oxidizes $\mathrm{OH}^{-}$to $\cdot \mathrm{OH}$ radicals, then the generated $\mathrm{h}^{+}$and $\cdot \mathrm{OH}$ degraded TC into other small molecules. Due to the modulated electronic structure of MA-LDH by Ti incorporation, more active sites on the surface were exposed and favored the formation of radicals, which promotes the catalytic reaction with enhanced kinetic feature. In addition, increased ability of visible light absorption provided more photoexcited carriers that participated in the redox reactions, and mesoporous nanostructure also promised more microchannels for mass and carrier transfer, which all contributes to the excellent photocatalytic activity for TC removal.

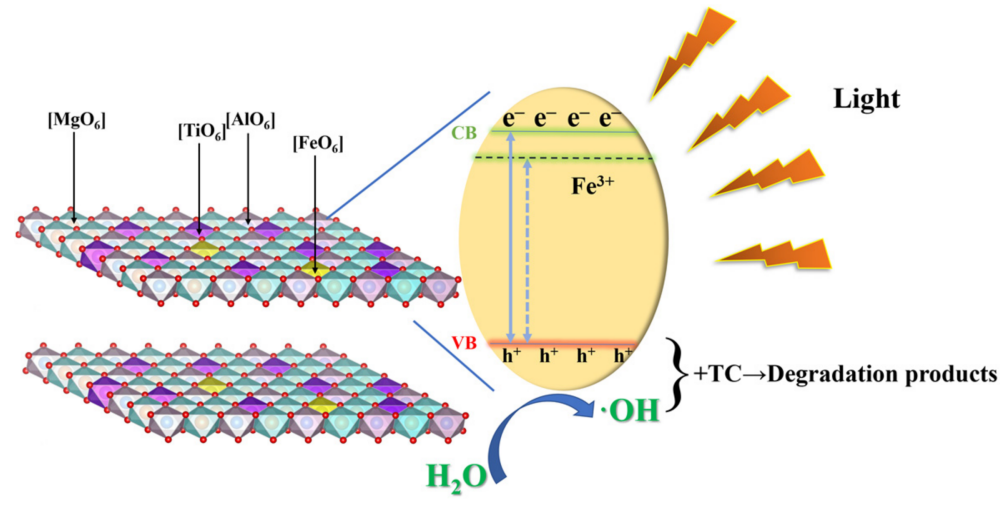

Figure 8. The mechanism of photo-degradation of TC by TMA-LDH under visible light.

\section{Conclusions}

In summary, we have demonstrated a heat treatment-precipitation strategy to efficient recycle Ti-containing blast furnace slag as visible-light-driven photocatalyst for TC removal. As indicated, Ti-embedded MgAl layered double hydroxide and Si-Ca-contained compound are obtained as products, which accounts for the overall recovery rate almost higher than $90 \%$. This recycling method also endows synthesized product with mesoporous structure and increased light response ability in visible range, and benefits the photocatalytic reaction. More importantly, controlled experiments suggest that Ti incorporation modifies the electronic structure of pristine LDH, which favors the formation of more available 
radicals and is responsible for the remarkably promoted photocatalytic activity. As a result, $88 \%$ degradation of initial concentration of TC could be achieved when adopting synthesized TMA-LDH as catalyst. The degradation efficiency is about 2.9 times higher than that of Ti-BFS, and a $44 \%$ increase compared to that of MA-LDH, respectively. Accordingly, as-proposed recycling strategy with high utilization efficiency addresses it as a feasible approach to the recovery of other slags with excellent properties for various applications.

Supplementary Materials: The following supporting information can be downloaded at: https: / / www.mdpi.com/article/10.3390/ma15041514/s1, Figure S1: (a) Low and (b) high magnified SEM images, (c) TEM images and (d) elemental mapping of obtained MA-LDH; Figure S2: Pore size distribution of obtained brucite products; Figure S3: XPS spectra of Fe 2p in Ti-BFS; Figure S4: Band gap energy of TMA-LDH and MA-LDH; Figure S5: XPS spectra of the carbon component; Table S1: Main composition of MA-LDH; Table S2: General weight ratios that converted CSS and TMA-LDH.

Author Contributions: Conceptualization, N.S. and Y.C.; methodology, N.S. and Q.Z.; validation, L.S.; formal analysis, N.S. and Y.C.; investigation, N.S. and Q.Z.; resources, J.W. (Jinshu Wang) and J.W. (Junshu $\mathrm{Wu}$ ); data curation, N.S.; writing—original draft preparation, N.S.; writing-review and editing, P.H.; visualization, P.H.; supervision, P.H. and J.W. (Jinshu Wang); project administration, J.W. (Jinshu Wang); funding acquisition, J.W. (Jinshu Wang) and J.W. (Junshu Wu). All authors have read and agreed to the published version of the manuscript.

Funding: This research was funded by the National Natural Science Foundation of China (Nos. 51974011, 52172290), and the National Natural Science Found for Innovative Research Groups (No. 51621003).

Institutional Review Board Statement: Not applicable.

Informed Consent Statement: Not applicable.

Data Availability Statement: Not applicable.

Conflicts of Interest: The authors declare that they have no known competing financial interests or personal relationships that could have appeared to influence the work reported in this paper.

\section{References}

1. Cai, Y.F.; Song, N.N.; Yang, Y.F.; Sun, L.M.; Hu, P.; Wang, J.S. Recent progress of efficient utilization of titanium-bearing blast furnace slag. Int. J. Min. Met. Mater. 2022, 29, 22-31. [CrossRef]

2. Zhang, Y.M.; Wang, L.N.; Chen, D.S.; Wang, W.J.; Liu, Y.H.; Zhao, H.X.; Qi, T. A method for recovery of iron, titanium, and vanadium from vanadium-bearing titanomagnetite. Int. J. Miner. Met. Mater. 2018, 25, 131-144. [CrossRef]

3. Gong, P.J.; Xie, J.L.; Wua, H.T.; Zhang, Y.P.; Cheng, X.K. Novel CeZrTiAl catalyst for $\mathrm{NH}_{3}-\mathrm{SCR}$ of $\mathrm{NO}_{\mathrm{x}}$ based on Ti-bearing BFS. J. Environ. Chem. Eng. 2021, 9, 105233. [CrossRef]

4. $\quad$ Dong, H.G.; Jiang, T.; Guo, Y.F.; Chen, J.L.; Fan, X.X. Upgrading a Ti-slag by a roast-leach process. Hydrometallurgy 2012, 113-114, 119-121. [CrossRef]

5. Safdar, F.; Zhang, Y.; Zheng, S.L.; Chen, X.; Sun, P.; Zhang, Y.; Li, P. Recovery of $\mathrm{TiO}_{2}$-enriched material from vanadium titano-magnetite concentrates by partial carbon reduction and mild acid leaching. Hydrometallurgy 2020, 193, 105324. [CrossRef]

6. Du, Y.; Gao, J.T.; Lan, X.; Guo, Z.C. Recovery of rutile from Ti-bearing blast furnace slag through phase transformation and super-gravity separation for dielectric material. Ceram. Int. 2020, 46, 9885-9893. [CrossRef]

7. Yang, D.; Zhou, H.H.; Wang, J.; Pang, Z.D.; Pei, G.S.; Yan, Z.M.; Mao, H.X.; Qiu, G.B.; Lv, X.W. Influence of TiO 2 on viscosity, phase composition and structure of chromium-containing high-titanium blast furnace slag. J. Mater. Res. Technol. 2021, 12, 1615-1622. [CrossRef]

8. Bian, X.A.; Zhang, S.; Zhao, Y.X.; Shi, R.; Zhang, T.R. Layered double hydroxide-based photocatalytic materials toward renewable solar fuels production. InfoMat 2021, 3, 719-738. [CrossRef]

9. Yang, W.W.; Cai, W.Q.; Zhou, J.X.; Dang, C.X.; Peng, X.; Chen, Y.T.; Wei, X.C.; Bo, S.L.; Liang, S.L.; Luo, Z.J. Mussel-inspired $\mathrm{MgAl}-\mathrm{LDH} /$ carbon fiber film modified by polydopamine for highly efficient removal of $\mathrm{Pb}^{2+}$. J. Environ. Chem. Eng. 2021, 9, 106634. [CrossRef]

10. Wu, Y.Y.; Yu, Y.; Zhou, J.Z.; Liu, J.Y.; Chi, Y.; Xu, Z.P.; Qian, G.R. Effective removal of pyrophosphate by Ca-Fe-LDH and its mechanism. Chem. Eng. J. 2012, 179, 72-79. [CrossRef]

11. Li, Y.W.; Gao, W.; Peng, M.; Zhang, J.B.; Sun, J.L.; Xu, Y.; Hong, S.; Liu, X.; Liu, X.W.; Wei, M.; et al. Interfacial Fe ${ }_{5} \mathrm{C}_{2}-\mathrm{Cu}_{\mathrm{c}}$ catalysts toward low-pressure syngas conversion to long-chain alcohols. Nat. Commun. 2020, 11, 61. [CrossRef]

12. Gao, Z.F.; Liang, J.X.; Yao, J.; Zhao, Y.T.; Meng, Q.; He, G.Y.; Chen, H.Q. Synthesis of Ce-doped NiAl LDH/RGO composite as an efficient photocatalyst for photocatalytic degradation of ciprofloxacin. J. Environ. Chem. Eng. 2021, 9, 105405. [CrossRef] 
13. Hu, J.; Tang, X.M.; Dai, Q.; Liu, Z.Q.; Zhang, H.M.; Zheng, A.M.; Yuan, Z.Z.; Li, X.F. Layered double hydroxide membrane with high hydroxide conductivity and ion selectivity for energy storage device. Nat. Commun. 2021, 12, 3409. [CrossRef]

14. Chen, H.; Hu, L.F.; Chen, M.; Yan, Y.; Wu, L.M. Nickel-cobalt layered double hydroxide nanosheets for high-performance supercapacitor electrode materials. Adv. Funct. Mater. 2014, 24, 934-942. [CrossRef]

15. Hoyo, C.D. Layered double hydroxides and human health: An overview. Appl. Clay Sci. 2007, 36, $103-121$.

16. Gao, R.; Mei, X.; Yan, D.P.; Liang, R.Z.; Wei, M. Nano-photosensitizer based on layered double hydroxide and isophthalic acid for singlet oxygenation and photodynamic therapy. Nat. Commun. 2018, 9, 2798. [CrossRef]

17. Dib, M.; Ouchetto, H.; Akhramez, S.; Fadili, H.; Essoumhi, A.; Ouchetto, K.; Hafid, A.; Sajieddine, M.; Khouili, M. Preparation of $\mathrm{Mg} / \mathrm{Al}-\mathrm{LDH}$ nanomaterials and its application in the condensation of 3-amino-1-phenyl-2-pyrazolin-5-one with aromatic aldehyde. Mater. Today 2020, 22, 104-107. [CrossRef]

18. Huang, C.; Nie, J.; Xu, Z.; Zhang, X.; Tang, J.; Wang, B.; Huang, J.; Du, C.; Chen, J. One-step hydrothermal synthesized 3D $\mathrm{P}-\mathrm{MoO}_{3} / \mathrm{FeCo} \mathrm{LDH}$ heterostructure electrocatalysts on $\mathrm{Ni}$ foam for high-efficiency oxygen evolution electrocatalysis. Int. J. Hydrog. Energy 2021, 46, 12992-13000. [CrossRef]

19. Kumari, P.; Das, R.K.; Pal, B. Enhanced photocatalytic degradation of eco-toxic pharmaceutical waste diclofenac sodium by anion loaded $\mathrm{Cu}-\mathrm{Al} \mathrm{LDH} \cdot \mathrm{Bi}_{2} \mathrm{O}_{3}$ composites. J. Taiwan Inst. Chem. Eng. 2021, 129, 227-236. [CrossRef]

20. Smalenskaite, A.; Vieira, D.E.L.; Salak, A.N.; Ferreira, M.G.S.; Ferreira, A.; Kareiva, A. A comparative study of co-precipitation and sol-gel synthetic approaches to fabricate cerium-substituted $\mathrm{Mg}$-Al layered double hydroxides with luminescence properties. Appl. Clay Sci. 2017, 143, 175-183. [CrossRef]

21. Xiao, L.P.; Li, Y.; Kong, Q.P.; Lan, Y.L. From wastes to functions: Preparation of layered double hydroxides from industrial waste and its removal performance towards phosphates. Environ. Sci. Pollut. Res. 2021, 29, 11893-11906. [CrossRef] [PubMed]

22. Pavela, O.; Stamatea, A.; Zăvoianua, R.; Bucurb, I.; Bîrjegac, R.; Angelescua, E.; Pârvulescua, V. Mechano-chemical versus co-precipitation for the preparation of Y-modified LDHs for cyclohexene oxidation and Claisen-Schmidt condensations. Appl. Catal. A Gen. 2020, 605, 117797. [CrossRef]

23. Zhang, P.; Qian, G.; Xu, Z.; Shi, H.; Ruan, X.; Yang, J.; Frost, R. Effective adsorption of sodium dodecylsulfate (SDS) by hydrocalumite (CaAl-LDH-Cl) induced by self-dissolution and re-precipitation mechanism. J. Colloid. Interf. Sci. 2012, 367, 264-271. [CrossRef] [PubMed]

24. Wang, L.; Xing, H.; Zhang, S.; Ren, Q.; Pan, L.; Zhang, K.; Bu, W.; Zheng, X.; Zhou, L.; Peng, W. A Gd-doped Mg-Al-LDH/Au nanocomposite for CT/MR bimodal imagings and simultaneous drug delivery. Biomaterials 2013, 34, 3390-3401. [CrossRef]

25. Zhang, Q.; Xu, R.; Liu, N.; Dai, C.; Yu, G.; Wang, N.; Chen, B. In situ Ce-doped catalyst derived from NiCeAl-LDHs with enhanced low-temperature performance for $\mathrm{CO}_{2}$ methanation. Appl. Surf. Sci. 2022, 579, 152204. [CrossRef]

26. Hadj-Abdelkader, N.E.H.; Beltrao-Nunesa, A.P.; Belkhademb, F.; Benselkab, N.; Roya, R.; Azzouz, A. New insights in MgAl and $\mathrm{MgFe}-\mathrm{LDH}$ affinity towards carbon dioxide-Role of the hydrophilic character on $\mathrm{CO}_{2}$ retention strength. Appl. Clay Sci. 2020, 198, 105829. [CrossRef]

27. Wu, X.L.; Tan, X.L.; Yang, S.T.; Wen, T.; Guo, H.L.; Wang, X.K.; Xu, A.W. Coexistence of adsorption and coagulation processes of both arsenate and NOM from contaminated groundwater by nanocrystallined $\mathrm{Mg} / \mathrm{Al}$ layered double hydroxides. Water Res. 2013, 47, 4159-4168. [CrossRef]

28. He, J.; Shi, H.M.; Shu, X.; Li, M.L. On the nature of Ti(IV)-pillared layered metal hydroxides prepared from green, water-soluble Ti-peroxide. AIChE J. 2010, 56, 1352-1362. [CrossRef]

29. Luo, X.G.; Lei, X.J.; Cai, N.; Xie, X.P.; Xue, Y.N.; Yu, F.Q. Removal of heavy metal ions from water by magnetic cellulose-based beads with embedded chemically modified magnetite nanoparticles and activated carbon. ACS Sustain. Chem. Eng. 2016, 4 , 3960-3969. [CrossRef]

30. Vyalikh, A.; Costa, F.R.; Wagenknecht, U.; Heinrich, G.; Massiot, D.; Scheler, U. From layered double hydroxides to layered double hydroxide-based nanocompositess-a solid-state NMR Study. J. Phys. Chem. C 2009, 113, 21308-21313. [CrossRef]

31. Licheron, M.; Montouillout, V.; Millot, F.; Neuville, D.R. Raman and ${ }^{27}$ Al NMR structure investigations of aluminate glasses: $(1-\mathrm{x}) \mathrm{Al}_{2} \mathrm{O}_{3-\mathrm{x}} \mathrm{MO}$, with $\mathrm{M}=\mathrm{Ca}, \mathrm{Sr}, \mathrm{Ba}$ and $\left.0.5<\mathrm{x}<0.75\right)$. J. Non-Cryst. Solids 2011, 357, 2796-2801.

32. Arco, M.; Fernández, A.; Martín, C.; Rives, V. Solubility and release of fenbufen intercalated in $\mathrm{Mg}, \mathrm{Al}$ and $\mathrm{Mg}, \mathrm{Al}, \mathrm{Fe}$ layered double hydroxides (LDH): The effect of eudragit ${ }^{\circledR}$ S 100 covering. J. Solid State Chem. 2010, 183, 3002-3009. [CrossRef]

33. Rahman, M.T.; Kameda, T.; Kumagai, S.; Yoshioka, T. A novel method to delaminate nitrate-intercalated Mg-Al layered double hydroxides in water and application in heavy metals removal from waste water. Chemosphere 2018, 203, 281-290. [CrossRef]

34. Du, X.C.; Huang, J.W.; Zhang, J.J.; Yan, Y.C.; Wu, C.Y.; Hu, Y.; Yan, C.Y.; Lei, T.Y.; Chen, W.; Fan, C.; et al. Modulating electronic structures of inorganic nanomaterials for efficient electrocatalytic water splitting. Angew. Chem. Int. Ed. 2019, 58, 4484-4502. [CrossRef]

35. Wu, Y.S.; Liu, X.J.; Han, D.D.; Song, X.Y.; Shi, L.; Song, Y.; Niu, S.W.; Xie, Y.F.; Cai, J.Y.; Wu, S.Y.; et al. Electron density modulation of $\mathrm{NiCO}_{2} \mathrm{~S}_{4}$ nanowires by nitrogen incorporation for highly efficient hydrogen evolution catalysis. Nat. Commun. 2018, 9, 1425. [CrossRef]

36. Yang, C.; Yuan, J.; Guo, Y.; Luo, X.G. In situ nano-assembly of Mg/Al LDH embedded on phosphorylated cellulose microspheres for tetracycline hydrochloride removal. Cellulose 2020, 28, 301-316. [CrossRef]

37. Lu, R.J.; Xu, X.; Chang, J.P.; Zhu, Y.; Xu, S.L.; Zhang, F.Z. Improvement of photocatalytic activity of TiO ${ }_{2}$ nanoparticles on selectively reconstructed layered double hydroxide. Appl. Catal. B Environ. 2012, 111-112, 389-396. [CrossRef] 
38. Duan, S.; Chen, S.Q.; Wang, T.Y.; Li, S.Z.; Liu, J.Y.; Liang, J.S.; Xie, H.Q.; Han, J.T.; Jiao, S.H.; Cao, R.G.; et al. Elemental selenium enables enhanced water oxidation electrocatalysis of NiFe layered double hydroxides. Nanoscale 2019, 11, 17376-17383. [CrossRef]

39. Wang, Z.C.; Fang, P.F.; Kumar, P.; Wang, W.W.; Liu, B.; Li, J. Controlled growth of LDH films with enhanced photocatalytic activity in a mixed wastewater treatment. Nanomaterials 2019, 9, 807. [CrossRef]

40. Gao, G.; Zhu, Z.; Zheng, J.; Liu, Z.; Wang, Q.; Yan, Y.S. Ultrathin magnetic Mg-Al LDH photocatalyst for enhanced $\mathrm{CO}_{2}$ reduction: Fabrication and mechanism. J. Colloid. Interf. Sci. 2019, 555, 1-10. [CrossRef]

41. Shi, Y.L.; Li, J.Q.; Zhang, B.Y.; Lv, S.Y.; Wang, T.; Liu, X. Tuning electronic structure of CoNi LDHs via surface Fe doping for achieving effective oxygen evolution reaction. Appl. Surf. Sci. 2021, 565, 150506. [CrossRef]

42. Khodam, F.; Amani-Ghadim, H.R.; Aber, S.; Amani-Ghadim, A.R.; Ahadzadeh, I. Neodymium doped mixed metal oxide derived from CoAl-layered double hydroxide: Considerable enhancement in visible light photocatalytic activity. J. Ind. Eng. Chem. 2018, 68, 311-324. [CrossRef]

43. Wang, X.Y.; Tuo, Y.X.; Zhou, Y.; Wang, D.; Wang, S.T.; Zhang, J. Ta-doping triggered electronic structural engineering and strain effect in NiFe LDH for enhanced water oxidation. Chem. Eng. J. 2021, 403, 126297. [CrossRef]

44. Lee, Y.; Choi, J.H.; Jeon, H.J.; Choi, K.M.; Lee, J.W.; Kang, J.K. Titanium-embedded layered double hydroxides as highly efficient water oxidation photocatalysts under visible light. Energy Environ. Sci. 2011, 4, 914-920. [CrossRef]

45. Zhou, M.; Han, D.; Liu, X.; Ma, C.; Wang, H.; Tang, Y.; Huo, P.; Shi, W.; Yan, Y.; Yang, J. Enhanced visible light photocatalytic activity of alkaline earth metal ions-doped CdSe/rGO photocatalysts synthesized by hydrothermal method. Appl. Catal. B Environ. 2015, 172-173, 174-184. [CrossRef]

46. Zhang, Q.; Jiang, L.; Wang, J.; Zhu, Y.; Pu, Y.; Dai, W. Photocatalytic degradation of tetracycline antibiotics using three-dimensional network structure perylene diimide supramolecular organic photocatalyst under visible-light irradiation. Appl. Catal. B Environ. 2020, 277, 119122. [CrossRef]

47. Jing, H.; Ou, R.; Yu, B.; Zhao, Y.; Lu, Y.; Huo, M.; Huo, H.; Wang, X. Engineering of g- $\mathrm{C}_{3} \mathrm{~N}_{4}$ nanoparticles/WO 3 hollow microspheres photocatalyst with Z-scheme heterostructure for boosting tetracycline hydrochloride degradation. Sep. Purif. Technol. 2021, 255, 117646. [CrossRef] 